\title{
IMPACT OF SOCIO-ECONOMIC VARIABLES AND TRAVEL ENVIRONMENT ON PERCEPTION OF TRAVEL TIME: A CASE STUDY OF SURAT, INDIA
}

\author{
Krishna Saw ${ }^{1}$, Bhimaji K. Katti ${ }^{2}$, Gaurang J. Joshi ${ }^{3}$ \\ ${ }_{1,2,3}$ Civil Engineering Department, SVNIT, Surat, Gujarat-395007, India
}

Received 16 June, 2016; accepted 22 September, 2016

\begin{abstract}
Transport planners consider the travel time as the most important design and performance criteria for comparison of routes or road segments in realizing urban travel efficiency. It is the decisive tool for traffic operation and improvement measures in urban transport system. However, Perceived Travel Time (PTT) has gained more importance over the Measured Travel Time in recent time, as it is associated with drivers' and commuters' expressions on their traffic and route situation analysis rather than the measured one. Moreover they are to take decision on their travel plan, mode, and route choice etc. on their perception basis only. The present study focusses on the effect of socio-economic variables and the travel environment on their decisions by a study on a major traffic corridor in Surat, Gujarat for both peak and off-peak situations. Interestingly the inference is that except income there is no significant impact on perception of Travel Time. However, the traffic congestion level and physical environment prevailing have significant bearing on their judgements.
\end{abstract}

Keywords: perceived travel time, socio-economic variables, travel environment, traffic congestion.

\section{Introduction}

Travel time is probably one of the most studied attributes in route choice, route performance or departure time analysis and modelling. In reality this is not the case because drivers may have different perception of travel time for particular route (Ramazani et al., 2011). Often travel experience does matter in travel time perception and differ from the actual travel time. Now, therefore, perception of travel time is receiving more attention in route choice modelling (Vreeswijk et al., 2014). There is significant difference between perceived and measured travel time. Mostly there is overestimate in perceived travel time on part of drivers. The perceived time tends to increase with discomfort, insecurity and congestion. Perceived time differs from driver to driver or commuter to commuter based on their judgement of traffic conditions, journey episodes, travel environment etc. It is equally important to note the influence of socio-economic variables along with the travel environment in travel time perception. With this objective, a study has been carried out on a major traffic corridor of Surat city, the second largest city in Gujarat state.

\footnotetext{
${ }^{1}$ Corresponding author: kriscivil_10@yahoo.com
} 


\section{Literature review}

Perceived travel time (PTT) has been defined as the driver's expected travel time on a trip before departing the origin (Lee et al., 2007). It is an estimated travel time of traveller according to Connors and Sumalee (2009) and Ramezani and Geroliminis (2012). Perceived travel time as per Li (2003) is a function of commute characteristics, journey episodes, travel environments and expectancy. Most of the researchers have opinion that the travellers take decision on the basis of perceived travel time rather than actual travel time. The decisions are based on drivers' experience (Poon and Stopher, 2011). The transport planners on the contrary make use of measured travel time (Ushiwaka, 2004; Kirpalani and Gelfend, 1980). However, Glob and Dosbson, 1974 considered PTT as the key variable in policy framework and not the actual travel time. Uncertainty of the traffic environment and travel time variability influence on perception of transit travel time (Chen et al., 2011). The uncertainty originates from varied perceptions of drivers. Parthasarathi et al. (2013) considers that the network variables such as street density, access roads, intersection, intensity etc. are equally important in such studies. Various socio-economic variables are incorporated in perceived travel time analysis by Peer et al. (2014). Departure time choice model is developed by Tseng et al. (2008) with reference to perceived travel time. Shortest time route and perceived shortest routes are part of route choice in the Dutch city in the study of Vreeswijk et al. (2014) in Trieste (Italy).

\section{Study Approach}

An arterial road $11 \mathrm{~km}$ in length that connects the two important areas of the city i.e. Dumas to city railway station is considered for the study. Physical characteristics such as number of lane, number of intersections, service road availability are noted and the same time encroached areas by vendors and parking is earmarked along the corridor. The physical characteristics are collected by dividing the whole section in three segments based on width uniformity of sections. Traffic volume and composition have been noted on three sections during peak and off peak period to know traffic characteristics. Perceived travel times of individual commuter is collected using predesigned question format using road side interview technique. Subsequently, data is analyzed for variations in PTT with respect to socioeconomic variables and travel environment. Statistical and ANOVA test is conducted to check tests to realize the influence of variables.

\section{Study Area and Corridor}

\subsection{City Background}

Surat city, the study area is a fast growing industrial metropolitan city in South Gujarat which has a historical base. It is the second largest city with a population of nearly 53 lac in the State and. It is known for its textile manufacturing, chemical industries, and as well diamond cutting and polishing, and intricate zari works. The gas based industries established by leading industry houses such as ONGC, Reliance, ESSAR, and Shell etc. are also in the vicinity. Having strong industrial base, urbanization of the city is of higher order to an extent of $55 \%+$ as decadal growth.

\subsection{Study Corridor}

The length of the selected corridor is approximately $11 \mathrm{~km}$ from Rahul Raj, a 
popular mall having significant number of consumers on one end and continue up-to Railway Station via Athwa gate-Ring Road (Fig. 1). The divide corridor consists of three signalized intersections at Choupati, Athwa-Gate and Sasu Maa and two roundabouts at Shahid Chowk and SVNIT gate.
The corridor passes through important residential, commercial, institutional areas. The District Court and airport are also situated on this corridor. The corridor is in radial form up to Athwa gate, followed by ring road up to railway station having number of flyovers.

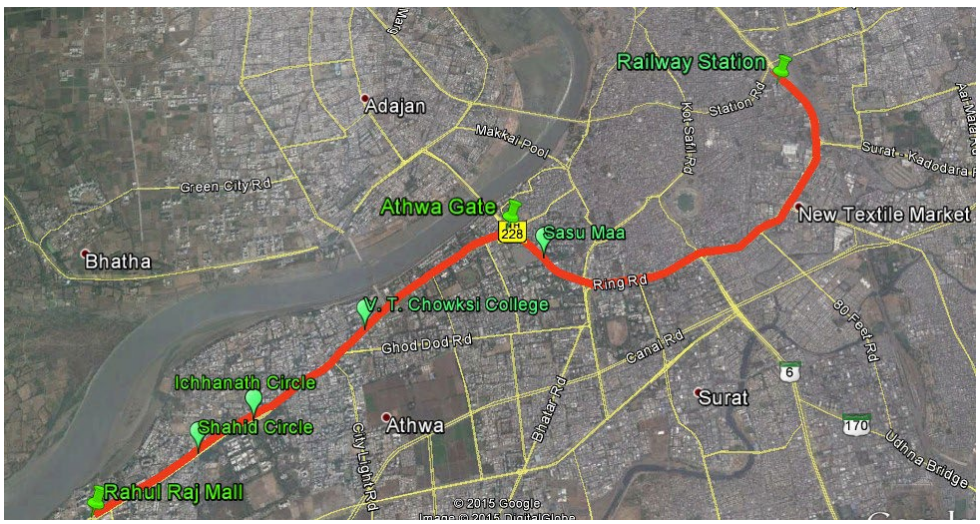

Fig. 1.

Study Corridor

\section{Surveys and Observations}

Two major field studies are carried: Traffic characteristics to note volume, composition and road side environment followed by socio-economic surveys and observations, perceived values on traffic congestion and travel time through roadside interviews, driver characteristics, perceived times. Classified volume count is conducted, one at each segment. The volume count has been conducted during peak and off peak period using video graphic technique to know the variation of the traffic and composition.

\subsection{Traffic Characteristics of Study Corridor}

On an average mixed traffic volume is 4500 5000 vehicles per hour in one direction. Composition wise two wheelers are predominating to an extent of $50 \%$ to $60 \%$. Traffic volume and composition particulars are briefed below.

Table 1

Average Traffic Volume (veh/hr) in up Direction

\begin{tabular}{|c|c|c|c|}
\hline \multirow{2}{*}{ Period } & \multicolumn{3}{|c|}{ Segment } \\
\cline { 2 - 4 } & 1 & 2 & 3 \\
\hline M. Peak & 3671 & 5686 & 4968 \\
\hline E. Peak & 3756 & 6721 & 5180 \\
\hline Off Peak & 3238 & 5048 & 4235 \\
\hline
\end{tabular}


Table 2

Traffic Composition

\begin{tabular}{|l|l|l|l|}
\hline 2Wheelers & 3Wheelers & CARS & Others \\
\hline $50-65 \%$ & $15-20 \%$ & $20-30 \%$ & $10-15 \%$ \\
\hline
\end{tabular}

With regard to traffic speed, speed is measured using two-wheeler as probe vehicle. The speed of probe vehicle during peak period is $28 \mathrm{kmph}$ whereas $35 \mathrm{kmph}$ during off peak period.

\subsection{Perceived Travel Time Surveys}

Perceived travel time of individual driver/ commuter is collected using predesigned questionnaire format by road side interview technique. The survey was conducted during peak and off peak period to capture variation in perceived travel time. Survey Format is in to three parts; travel characteristics, socioeconomic characteristics, and perceived travel time with congestion level and environmental condition. The sample sizes are of 300 and 250 for peak and offpeak periods respectively. The questionnaire covers:
- Trip Particulars -the trip purpose, journey starting time and frequency of journey

- Commuters profile - socioeconomic characteristics such as Gender, Age, Income, Education and Occupation etc.

- Commuters' travel time perceptions.

- Respondents were asked their perception on Journey time in peak and off peak period. The congestion levels and environment condition in five linguistic expressions: Very Low, Low, Medium, High and Very High levels.

\section{Perceived Travel Time Analysis}

\subsection{Statistical Measures}

Statistical measures of Perceived Travel Time for the route during peak and off-peak periods are summarized for their deviations and average values (Table 3 ).

\section{Table 3}

Statistical Measures*

\begin{tabular}{|l|l|l|}
\hline Parameters & Peak & Off Peak \\
\hline Min Time & 15 & 10 \\
\hline Max Time & 60 & 45 \\
\hline Std. Dev & 10.40 & 9.95 \\
\hline Average & 39.63 & 26.17 \\
\hline
\end{tabular}

* in minutes

Average PTT of users during peak period is 40 minutes and during off peak it is 26 minutes. Standard deviation is higher in peak period. The PTT data of peak and off-peak was found to follow normal distribution as per chi-square criteria at $(\alpha=$ $0.05)$. Frequency curves for peak and off- peak observed normal distribution based are shown in Fig 2. Expected frequency is derived using normal probability distribution function as observed PTT data follows normal distribution. The PTT spread is more for peak period reflecting on over and under estimation of travel time. 


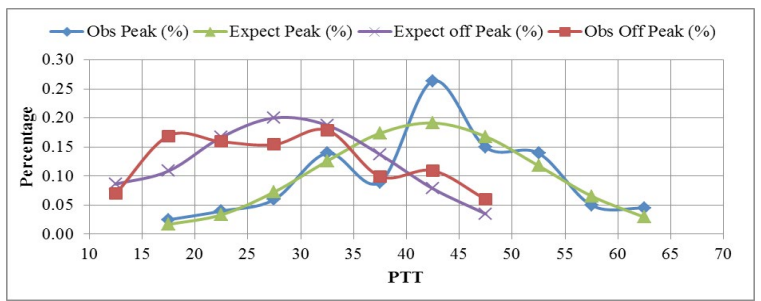

Fig. 2.

Observed and Expected Frequency during Peak and Off Peak

\subsection{Socio-economic Attributes and PTT Variations}

Observations of Perceived Travel Time on the basis of socioeconomic characteristics are mentioned in Table 4. The main attributes considered are gender, age, income and education. Influence of income level, age and education has been assessed. Higher income group has indicated lower PTT value compared with both low and middle income groups. Age wise PTT values are increasing order with the age. As far as education is considered, higher PTT for low education with reference to graduate riders. Education and gender have shown hardly any difference.

Table 4

Socioeconomic Characteristics Influence on PTT*

\begin{tabular}{|c|c|c|c|c|c|c|}
\hline \multicolumn{2}{|c|}{ Attribute } & $\begin{array}{c}\text { Avg Peak Period } \\
\text { (PTT })\end{array}$ & PTT $_{\text {max }} /$ & Off Peak Period & $\mathbf{P T T}_{\text {max }} /$ & $\mathbf{P T T}_{\mathrm{p}} / \mathbf{P T T}_{\mathrm{o}}$ \\
\hline \multirow{2}{*}{ Gender } & Male & 40 & \multirow{2}{*}{1.05} & 29 & \multirow{2}{*}{1.07} & 1.38 \\
\hline & Female & 38 & & 27 & & 1.41 \\
\hline \multirow{3}{*}{ Income } & Low Income & 42 & \multirow{3}{*}{1.35} & 32 & \multirow{3}{*}{1.52} & 1.31 \\
\hline & Medium Group & 43 & & 31 & & 1.39 \\
\hline & High Group & 31 & & 21 & & 1.48 \\
\hline \multirow{3}{*}{ Age } & Young & 38 & \multirow{3}{*}{1.18} & 29 & \multirow{3}{*}{1.10} & 1.31 \\
\hline & Middle & 41 & & 30 & & 1.37 \\
\hline & Elders & 45 & & 32 & & 1.41 \\
\hline \multirow{2}{*}{ Education } & Graduation & 40 & \multirow{2}{*}{1.08} & 28 & \multirow{2}{*}{1.07} & 1.43 \\
\hline & Others & 43 & & 30 & & 1.43 \\
\hline
\end{tabular}

* in minutes

PTT ratios presented in the table above reflect that ratio is only for the income factor. The ratios are marginally varying for other attributes.

The ratio values are quite significant for all the attributes in the range of 1.31 to 1.48 to reflect that the observations of PTT for both the periods are quite distinct and the values are set by chance.

\subsection{Travel Environment and PTT Variations}

\subsubsection{Trip Purpose}

From Table 5, it is observed that there is no significant variation in PTT with respect to trip purpose. However, PTT is less in case of work trips because this is performed daily and hence estimation is in lower side. 
Table 5

Purpose Wise PTT (minutes)

\begin{tabular}{|l|l|l|}
\hline Purpose & PTT -Peak & PTT - Off Peak \\
\hline Rly. Station Trip & 40 & 26 \\
\hline Social Activity & 40 & 28 \\
\hline Work & 38 & 25 \\
\hline
\end{tabular}

\subsubsection{Traffic Congestion}

Traffic congestion influences perceived travel time to a large extent. Respondents were asked level of congestion along with PTT (min), categorized in four groups,
Lower, Medium, High and Very High. The result is as shown in Fig 3. It is observed that as congestion level increases PTT also increases. The variation is from average $23 \mathrm{~min}$ to average $41 \mathrm{~min}$ for very high congestion situation.

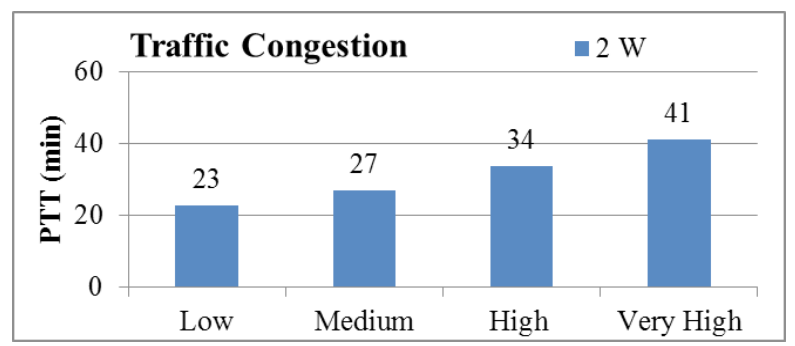

Fig. 3.

Traffic Congestion and PTT

\subsubsection{Environment Conditions}

Environmental condition is defined as overall feeling towards pollution, pedestrian encroachment, road side and parking disturbance etc. The attributes are rated by the travelers in five grades from $A$ to $E$ quality wise. (A - Very good, B - Good, C - Fair, D Poor, E - Very poor). PTT values are analyzed as below. D \& E levels are during peak period.

\section{Table 6}

Environmental Based PTT

\begin{tabular}{|l|l|l|l|l|l|}
\hline Environmental Condition & A & B & C & D & E \\
\hline PTT (min) & - & 24 & 28 & 38 & 42 \\
\hline
\end{tabular}

\section{Attribute Impact Analysis}

Here, ANOVA test has been carried to realize the influence of various variables on perception of travel time for peak and off peak periods. Analysis particulars against the attributes are provided in Table 6(a) and Table 6(b). Null hypothesis is rejected, if sample $f$ value is greater than $\mathrm{f}$ critical (threshold) otherwise accepted. Results indicate that, no significant effect is found for gender, age, education etc. except ( $\alpha$ $=0.05$ ) for income, traffic congestion, environmental factors. In Null hypothesis 
no much variation in $\mathrm{PTT}$ has been accepted but that is not the case of traffic congestion, income and environment. Similar observations are made by Peer et al. (2014). As far as purpose is concerned, no influence has been observed during the peak period and marginal influence is during the off peak period.

Table 6(a)

ANOVA Test Result for Peak Period

\begin{tabular}{|l|l|l|l|l|l|l|}
\hline Variable & Value & Sample F value & df & F Critical (threshold) & P Value & Remarks \\
\hline Gender & Male/Female & 0.007 & 1 & 3.945 & 0.934 & Accepted \\
\hline Age & $18-30 / 30-45 / 45-60 />60$ & 0.404 & 3 & 2.71 & 0.75 & Accepted \\
\hline Income & $<15,15-30,30-50,>50$ & 5.424 & 3 & 2.707 & 0.002 & Rejected \\
\hline Education & Graduate, Matric, Non-Matric & 0.271 & 2 & 3.097 & 0.763 & Accepted \\
\hline Occupation & Business, Govt., Pvt., Student & 1.17 & 3 & 2.707 & 0.325 & Accepted \\
\hline Purpose & Work/Social/Rly Station & 1.016 & 2 & 3.027 & 0.363 & Accepted \\
\hline Congestion* & Low/Medium/High/VH & 70.697 & 3 & 2.627 & 0 & Rejected \\
\hline Environmental* & Good/Fair/Poor/Very Poor & 53.499 & 3 & 2.627 & 0 & Rejected \\
\hline${ }^{*}$ Both Peak and off Peak & & & & & \\
\hline
\end{tabular}

Table 6(b)

ANOVA Test Result for off Peak Period

\begin{tabular}{|l|l|l|l|l|l|l|}
\hline Variable & Value & Sample F value & df & F Critical (threshold) & P Value & Remarks \\
\hline Gender & Male/Female & 0.007 & 1 & 3.945 & 0.934 & Accepted \\
\hline Age & $18-30 / 30-45 / 45-60 />60$ & 0.426 & 3 & 2.71 & 0.734 & Accepted \\
\hline Income & $<15,15-30,30-50,>50$ & 8.898 & 3 & 2.707 & 0 & Rejected \\
\hline Education & Graduate, Matric, Non-Matric & 0.877 & 2 & 3.097 & 0.419 & Accepted \\
\hline Occupation & Business, Govt., Pvt., Student & 1.331 & 3 & 2.707 & 0.269 & Accepted \\
\hline Purpose & Work/Social/Rly Station & 3.199 & 2 & 3.027 & 0.042 & Rejected \\
\hline
\end{tabular}

\section{Traffic Congestion and Environmental Impact}

\subsection{Sensing Traffic Quality}

ANOVA test reflects that traffic conditions on the road, significantly influence the perceived travel time of drivers/commuters.

- Uncertainty and Variability in traffic volume and composition

- Feeling of too many vehicles surrounding as a scenario

- Stuck up movement -The perception of worsening traffic stemmed in large part from having been stuck in traffic for significant lengths of time at bottlenecks and intersection.

- Frustration due to stop and go conditions during commute

These conditions have significant bearing on drivers' assessment of traffic quality varying from person to person and hence the higher PTT in comparison of measured travel time.

\subsection{Physical Environment}

ANOVA test reflects that environmental condition significantly influences the perceived travel time of road users/ commuters. Environmental condition 
comprises of mainly Roadside disturbance due to pedestrian encroachment, illegal parking and hackers etc. their judgements are based on overall situation along the route and do influence the drivers' physiological assessment.

\section{Conclusions}

Route performance assessment can be related eventually to travel time. Though measured Travel Time is mostly considered by the traffic and transportation engineers, it may be preferred only to take up to control the traffic hurdles on immediate basis of the route on physical ground. The Perceived Travel Time on the contrary, has been considered more important and now they are preferred as they are the expressions of the actual users who opt for route and mode choices for their travel planning. Lot of variations is observed in PTT values which need to set them in right perspective. To understand the route performance and required mitigation measures in order to raise the traffic speeds. Interestingly, it is found in the present study that the social variables of age, gender and education are not that significant. Similarly is the case of trip purposes. The main attributes identified for the perceived travel time are prevailing traffic congestion levels, an intensity of physical environmental disturbances along the route.

\section{References}

Chen, C.; Xu, Y.; Fu, X. 2011. Bus Route Travel Time Reliability Analysis Based on Travelers' Perceptions. In Proceedings of ICTE 2011, 352-357.

Connors, R.D.; Sumalee, A. 2009. A network equilibrium model with travellers' perception of stochastic travel times, Transportation Research Part B: Methodological, 43(6): 614-624.
Kirpalani, V.H.; Gelfand, L. 2015. Automobile Curtailment: Interaction with Psychological Variables/ Consequences/Strategies. In Marketing Horizons: A 1980's Perspective, 10-13. Springer International Publishing.

Lee S.; Moon B.; Lim K.; 2007. Evaluation of User Response to Reliable Shortest Path Information. In Proceedings of Eastern Asia Society of Transportation Studies, 1:87-95.

Li, Y.W.2003. Evaluating the urban commute experience: A time perception approach, Journal of Public Transportation, 6(4): 3p.

Parthasarathi, P., Levinson, D.; Hochmair, H., 2013. Network structure and travel time perception, PloS one, $8(10)$ : p.e77718.

Peer, S.; Knockaert, J.; Koster, P.; Verhoef, E.T. 2014. Over-reporting vs. overreacting: Commuters' perceptions of travel times, Transportation Research Part A: Policy and Practice, 69: 476-494.

Poon J. F.; Stopher P. R. 2011. Investigating the Effects of Different Types of Travel Information on Travellers' Learning in a Public Transport Setting using An Experimental Approach. In Proceedings of Australasian Transport Research Forum, $16 \mathrm{p}$.

Ramazani, H.; Shafahi, Y.; Seyedabrishami, S.E. 2011. A fuzzy traffic assignment algorithm based on driver perceived travel time of network links, Scientia Iranica, 18(2): 190-197.

Ramezani, M.; Geroliminis, N. 2012. On the estimation of arterial route travel time distribution with Markov chains, Transportation Research Part B: Methodological, 46(10): 1576-1590.

Tseng, Y.Y.; Verhoef, E.; de Jong, G.; Kouwenhoven, M.; van der Hoorn, T. 2009. A pilot study into the perception of unreliability of travel times using in-depth interviews, Journal of Choice Modelling, 2(1): 8-28. 
Ushiwaka, K.; Kikuchi, A.; Kitamura, R. 2004. Commuters' perception of travel time and uncertainty under congestion pricing: Exploration of a six-week field experiment data. In Proceedings of International Conference Experiments Economic Science, 922-938.

Vreeswijk, J.; Thomas, T.; van Berkum, E.; van Arem, B.; 2014. Perception bias in route choice, Transportation, 41(6): 1305-1321. 